\title{
IMPLICITIZATION OF TENSOR PRODUCT SURFACES IN THE PRESENCE OF A GENERIC SET OF BASEPOINTS
}

\author{
ELIANA DUARTE
}

\begin{abstract}
Given a 4-dimensional vector subspace $U=\left\{f_{0}, \ldots, f_{3}\right\}$ of $H^{0}\left(\mathbb{P}^{1} \times \mathbb{P}^{1}, \mathcal{O}(a, b)\right)$, a tensor product surface, denoted by $X_{U}$, is the closure of the image of the rational map $\lambda_{U}: \mathbb{P}^{1} \times \mathbb{P}^{1} \rightarrow \mathbb{P}^{3}$ determined by $U$. These surfaces arise in geometric modeling and in this context it is useful to know the implicit equation of $X_{U}$ in $\mathbb{P}^{3}$. In this paper we show that if $U \subseteq H^{0}\left(\mathbb{P}^{1} \times \mathbb{P}^{1}, \mathcal{O}(a, 1)\right)$ has a finite set of $r$ basepoints in generic position, then the implicit equation of $X_{U}$ is determined by two syzygies of $I_{U}=\left\langle f_{0}, \ldots, f_{3}\right\rangle$ in bidegrees $\left(a-\left\lceil\frac{r}{2}\right\rceil, 0\right)$ and $\left(a-\left\lfloor\frac{r}{2}\right\rfloor, 0\right)$. This result is proved by understanding the geometry of the basepoints of $U$ in $\mathbb{P}^{1} \times \mathbb{P}^{1}$. The proof techniques for the main theorem also apply when $U$ is basepoint free.
\end{abstract}

\section{Contents}

1. Introduction

2. Points in $\mathbb{P}^{1} \times \mathbb{P}^{1}$

3. Ideal of a generic set of points in $\mathbb{P}^{1} \times \mathbb{P}^{1}$

4. Syzygies of the ideal $I_{U}$

5. Applications to implicitization of tensor product surfaces

References

\section{INTRODUCTION}

Given a parameterized curve or surface in projective space, the implicitization problem consists on finding the equations whose vanishing is the closed image of the given parameterization. The implicitization problem has been of increasing interest to commutative algebraists and algebraic geometers due to its applications in Computer Aided Geometric Design(CAGD). In this context, knowing the implicit equation of the curve or surface is important to perform elementary operations with these objects. For example describing the curve of intersection of two surfaces, or the points of intersection of a curve and a 
surface. Equally important is the problem of testing if a given point in the codomain lies in the image of a parameterization. Using the implicit equations of a curve or surface to perform these operations is computationally and theoretically more efficient than only working with the parameterizations. For this reason, there is great interest in finding faster algorithms that compute implicit equations of parameterized curves and surfaces.

There are several general techniques to solve the implicitization problem for parameterized surfaces. These include Gröbner bases, resultants and syzygies. To summarize, Gröbner bases algorithms provide a straightforward theoretical approach that in practice tends to be very slow. Resultants provide a more convenient representation of the implicit equation as a determinant of a matrix but they fail for parameterizations with basepoints. In contrast, since their first appearance in the work of Sederberg and Chen [15] and Cox [8], syzygies have provided faster methods to obtain implicit equations that work for more general parameterizations. We elaborate more on these methods and compare timings for some examples in Section 5 .

We now focus on the implicitization problem for tensor product surfaces. Given a 4 dimensional vector space $U=\left\{f_{0}, \ldots, f_{3}\right\} \subset H^{0}\left(\mathbb{P}^{1} \times \mathbb{P}^{1}, \mathcal{O}(a, b)\right)$ with $\operatorname{gcd}\left(f_{0}, \ldots, f_{3}\right)=1$, we obtain a rational map $\lambda_{U}: \mathbb{P}^{1} \times \mathbb{P}^{1} \rightarrow \mathbb{P}^{3}$. A tensor product surface is the closure of the image of $\lambda_{U}$ and is denoted $X_{U}$. In this paper we use syzygy techniques to obtain the implicit equation of a tensor product surface $X_{U}$. One of the main tools of this approach is the approximation complex $\mathcal{Z}$ defined in Section 5. Approximation complexes were first introduced by Herzog, Simis and Vasconcelos [13] to study the defining equations of Rees algebras of ideals. An introduction to the use of these complexes to obtain implicit equations of parameterized hypersurfaces and the connection with Rees algebras can be found in Chardin's [5] work. These techniques were generalized for multigraded hypersurfaces such as tensor product surfaces by Botbol [1]. In Section 5 we use Botbol's results to obtain the implicit equation of $X_{U}$ via $\mathcal{Z}$.

In short, to find the implicit equation of $X_{U}$ using $\mathcal{Z}$ we fix a basis and find the matrices representing the maps of $\mathcal{Z}$ in a suitable degree $\nu$. Finally, the determinant of the complex $\mathcal{Z}_{\nu}$ is a power of the implicit equation of $X_{U}$. It is important to point out that for most practical purposes in CAGD, knowing the matrix of the map $d_{1}:\left(\mathcal{Z}_{\nu}\right)_{1} \rightarrow\left(\mathcal{Z}_{\nu}\right)_{0}$ is sufficient to perform the aforementioned elementary operations with curves and surfaces, for example as shown by Buse and Luu Ba [4] for the intersection of two surfaces. The matrix $d_{1}$ is known as a representation matrix for $X_{U}$. Representation matrices are generically of full rank and the gcd of its maximal minors is equal to a power of the implicit equation of 
$X_{U}$. These matrices have been studied by Botbol and Dickenstein [3] and Botbol, Buse and Chardin [2] among others.

The connection of $\mathcal{Z}$ with the syzygies of $U=\left\{f_{0}, \ldots, f_{3}\right\}$ comes from the observation that $\mathcal{Z}_{1}=\operatorname{Syz}\left(f_{0}, \ldots, f_{3}\right) \otimes S$, where $S:=k[X, Y, Z, W]$ is the coordinate ring of $\mathbb{P}^{3}$. Thus to obtain the matrices in $\mathcal{Z}_{\nu}$ we are led to the computation of $\operatorname{Syz}\left(f_{0}, \ldots, f_{3}\right)$. Let $I_{U}=\left\langle f_{0}, \ldots, f_{3}\right\rangle$ be the ideal generated by $U$ inside the total coordinate ring of $\mathbb{P}^{1} \times \mathbb{P}^{1}$. The vanishing locus $\mathbb{V}\left(I_{U}\right)$ inside $\mathbb{P}^{1} \times \mathbb{P}^{1}$ is referred to as the set of basepoints of $U$ and is denoted by $X$. If $X \neq \emptyset$ we say $U$ has basepoints, otherwise we say $U$ is basepoint free. We also refer to $X$ as the base locus of $\lambda_{U}$. The content of this paper follows the ideas from Duarte and Schenck [10] and of Schenck, Seceleanu and Validashti [14] in which the goal is to understand the syzygies that determine $\mathcal{Z}_{\nu}$. We focus on tensor product surfaces $X_{U}$ such that $U \subset H^{0}\left(\mathbb{P}^{1} \times \mathbb{P}^{1}, \mathcal{O}(a, b)\right)$ has $b=1$ and such that $X$ is a finite set of generic points.

Tensor product surfaces for which $b=1$ are also known as rational ruled surfaces in the literature. These surfaces have been studied before by Chen, Zheng and Sederberg [7] and by Dohm [9]. Chen, Zheng and Sederberg show that the implicit equation of a generically injective parameterization can be obtained from a submodule of the module of syzygies of $U$ and show that this module is of rank two. Dohm generalizes these results for a parameterization of any degree and provides an algorithm to reparameterize the given rational ruled surface. In this work we study the syzygies of $U$ based on the geometry of its base locus $X$. This approach gives the exact degrees of the syzygies that determine $\mathcal{Z}_{\nu}$ and answers the question raised by Chen, Cox and Liu [6] of what can be said about the degrees of the syzygies that determine the implicit equation. The statement of the main theorem is the following:

Theorem 1.1. Let $\left(I_{X}\right)_{(a, 1)} \subset H^{0}(a, 1)$ be the $k$-vector space of forms of bidegree $(a, 1)$ that vanish at a generic set $X$ of $r$ points in $\mathbb{P}^{1} \times \mathbb{P}^{1}$. Take $U=\left\{f_{0}, \ldots, f_{3}\right\}$ to be a generic 4-dimensional vector subspace of $\left(I_{X}\right)_{(a, 1)}$ and $\lambda_{U}: \mathbb{P}^{1} \times \mathbb{P}^{1} \rightarrow \mathbb{P}^{3}$ the rational map determined by $U$. Then the first map of the approximation complex $\mathcal{Z}$ in bidegree $\nu=(2 a-1,0)$, $\left(d_{1}\right)_{\nu}$, is determined by the syzygies of $\left(f_{0}, \ldots, f_{3}\right)$ in bidegrees $\left(a-\left\lfloor\frac{r}{2}\right\rfloor, 0\right), \quad\left(a-\left\lceil\frac{r}{2}\right\rceil, 0\right)$. The map $\left(d_{1}\right)_{\nu}$ completely determines the approximation complex $\mathcal{Z}$ in degree $\nu$ from which we compute the implicit equation of $X_{U}$.

Using the description of some of the minimal generators the ideal $I_{X}$ associated to a generic set of points $X$ in $\mathbb{P}^{1} \times \mathbb{P}^{1}$ given by Van Tuyl [17] we are able to understand exactly the degrees of the syzygies of $\left(f_{0}, \ldots, f_{3}\right)$ that determine the implicit equation of $X_{U}$. The subsequent sections of this paper are organized as follows. In Section 2 we introduce basic 
notation and several tools to study points in $\mathbb{P}^{1} \times \mathbb{P}^{1}$. In Section 3 we use results of Van Tuyl [17] to describe the minimal generators of the bihomogeneous ideal $I_{X}$ associated to a set $X$ of points in $\mathbb{P}^{1} \times \mathbb{P}^{1}$ in generic position. In Section 4 we use the results from Section 3 to describe the syzygies of the ideal $I_{U}$ where $U \subset\left(I_{X}\right)_{(a, 1)}$ is a generic four dimensional vector subspace. Finally in Section 5 we use the results from the previous sections to prove the main theorem for implicitization of tensor product surfaces in the presence of a generic set of basepoints by using the complex $\mathcal{Z}$ and give some examples. Throughout this paper $k$ will denote an algebraically closed field of characteristic zero.

\section{Points In $\mathbb{P}^{1} \times \mathbb{P}^{1}$}

The idea to prove Theorem 1.1 is to study the geometry of the base locus of $U$ in $\mathbb{P}^{1} \times \mathbb{P}^{1}$, to do this we focus on the ideals of $R$ that correspond to generic points in $\mathbb{P}^{1} \times \mathbb{P}^{1}$. In this section we describe such ideals and state a theorem of Van Tuyl for their bigraded Hilbert functions.

2.1. Notation. Let $R=\bigoplus_{0 \leq a, 0 \leq b} H^{0}\left(\mathbb{P}^{1} \times \mathbb{P}^{1}, \mathcal{O}(a, b)\right)$ be the total coordinate ring of $\mathbb{P}^{1} \times \mathbb{P}^{1}$. For shorter notation we write $H^{0}(a, b)$ for $H^{0}\left(\mathbb{P}^{1} \times \mathbb{P}^{1}, \mathcal{O}(a, b)\right)$. The ring $R$ is a bigraded $k$-algebra by taking $R_{(a, b)}=H^{0}(a, b)$ and $R$ is generated as a $k$-algebra by $H^{0}(1,0)$ and $H^{0}(0,1)$. Note that $\operatorname{dim} H^{0}(1,0)=\operatorname{dim} H^{0}(0,1)=2$. If $\{s, t\}$ is a basis for $H^{0}(1,0)$ and $\{u, v\}$ a basis for $H^{0}(0,1)$, then $R \cong k[s, t] \otimes k[u, v]$ with grading given by $\operatorname{deg} s, t=(1,0)$ and $\operatorname{deg} u, v=(0,1)$. An element $F \in R$ is bihomogeneous if $F \in R_{(i, j)}$ for some $(i, j) \in \mathbb{N}$. If $F$ is bihomogeneous, we say that its bidegree is $\operatorname{deg} F=(i, j)$. Suppose that $I=\left(F_{1}, \ldots, F_{n}\right) \subset R$ is an ideal. If each $F_{i}$ is bihomogeneous, then we say that $I$ is a bihomogeneous ideal.

For $U=\left\{f_{0}, \ldots, f_{3}\right\} \subset H^{0}(a, 1)$ we let $I_{U}:=\left\langle f_{0}, \ldots, f_{3}\right\rangle$ be the bihomogeneous ideal of $R$ generated by the elements of $U$.

2.2. Description of sets of points in $\mathbb{P}^{1} \times \mathbb{P}^{1}$. We follow the notation and definitions from Giuffrida, Maggioni and Ragusa [11] and from Guardo and Van Tuyl [12] to describe sets of points in $\mathbb{P}^{1} \times \mathbb{P}^{1}$. A point in $\mathbb{P}^{1} \times \mathbb{P}^{1}$ is a pair $A \times B$, where $A \in \mathbb{P}^{1}$ and $B \in \mathbb{P}^{1}$. Let $h$ be a non-zero linear form in the variables $s, t$ that vanishes at $A$ and let $l$ be a non-zero linear form in the variables $u, v$ that vanishes at $B$. The ideal of $R$ that corresponds to $P$ is denoted by $I_{P}$ and $I_{P}=\langle h, l\rangle$. We think of the form $h$ as a $(1,0)$ line and of $l$ as a $(0,1)$ line in $\mathbb{P}^{1} \times \mathbb{P}^{1}$. These lines are members of the two different rulings of $\mathbb{P}^{1} \times \mathbb{P}^{1}$ and a point in $\mathbb{P}^{1} \times \mathbb{P}^{1}$ is uniquely determined by their intersection. If $X=\left\{P_{1}, \ldots, P_{r}\right\}$ is a 
set of $r$ distinct points and $I_{P_{i}}=\left\langle h_{i}, l_{i}\right\rangle$, then $I_{X}$, the ideal corresponding to $X$, is given by $I_{X}=\bigcap_{i=1}^{r} I_{P_{i}}$.

There are two projections $\pi_{i}: \mathbb{P}^{1} \times \mathbb{P}^{1} \rightarrow \mathbb{P}^{1}$ defined by $\pi_{1}(A \times B)=A$ and $\pi_{2}(A \times B)=B$. We can visualize points in $\mathbb{P}^{1} \times \mathbb{P}^{1}$ as lying inside a grid of $(1,0)$ and $(0,1)$ lines.

From now on, $(1,0)$ lines will be drawn horizontally and $(0,1)$ lines will be drawn vertically. Throughout this paper, $X$ will denote a finite set of points in $\mathbb{P}^{1} \times \mathbb{P}^{1}$ and $I_{X}$ will denote its corresponding defining ideal in $R$.

As with sets of points in $\mathbb{P}^{n}$, we use Hilbert functions to study sets of points in $\mathbb{P}^{1} \times \mathbb{P}^{1}$. Since the ring $R$ is bigraded, the Hilbert function of $X$ takes the shape of a matrix.

Definition 2.1. Let $X$ be a finite set of points in $\mathbb{P}^{1} \times \mathbb{P}^{1}$. The bigraded Hilbert function of $X, H_{X}: \mathbb{Z} \times \mathbb{Z} \rightarrow \mathbb{N}$ is defined by

$$
H_{X}(i, j)=\operatorname{dim}_{k} R_{(i, j)}-\operatorname{dim}_{k}\left(I_{X}\right)_{(i, j)} .
$$

The bigraded Hilbert function of $X$ has similar properties to the Hilbert function of a set of points in projective space. When $\mathbb{P}^{1} \times \mathbb{P}^{1}$ is considered as a subvariety of $\mathbb{P}^{3}$ by the Segre embedding, $X$ becomes a subscheme of $\mathbb{P}^{3}$, in this case, $H_{X}(i)=H_{X}(i, i)=\operatorname{deg} X$ for all $i \gg 0$. The work of Guardo and Van Tuyl [12] provides a thorough introduction to the study of bigraded Hilbert functions of points in $\mathbb{P}^{1} \times \mathbb{P}^{1}$.

2.3. Combinatorial description of sets of points in $\mathbb{P}^{1} \times \mathbb{P}^{1}$. Using the description of a single point in $\mathbb{P}^{1} \times \mathbb{P}^{1}$ as the intersection of a $(1,0)$ line and a $(0,1)$ line, a set of points in $\mathbb{P}^{1} \times \mathbb{P}^{1}$ can be visualized as markings of some intersection points inside a rectangular grid as in Example 2.4. We describe sets of points in a combinatorial way using partitions. Let $h_{1}, \ldots, h_{q}$ be the horizontal lines in $\mathbb{P}^{1} \times \mathbb{P}^{1}$ that contain points of $X$. The ordering of these lines doesn't play any role so we may arrange them in such a way that

$$
\left|X \cap h_{1}\right| \geq\left|X \cap h_{2}\right| \geq \ldots \geq\left|X \cap h_{q}\right| \text {. }
$$

Let $\alpha_{i}=\left|X \cap h_{i}\right|$ and associate to $X$ the tuple $\alpha_{X}=\left(\alpha_{1}, \ldots, \alpha_{q}\right)$. Note that $\alpha_{X}$ is a partition of $|X|$. An analogous process yields a partition $\beta_{X}$ from $X$, which depends on the points on the vertical rulings of $X$. For the next theorem, we use the notion of the conjugate of a partition.

Definition 2.2. The conjugate of a partition $\lambda$ is the tuple $\lambda^{*}=\left(\lambda_{1}^{*}, \ldots, \lambda_{\lambda_{1}}^{*}\right)$ where $\lambda_{i}^{*}=\#\left\{\lambda_{j} \in \lambda \mid \lambda_{j} \geq i\right\}$ 
For a set $X$ of points in $\mathbb{P}^{1} \times \mathbb{P}^{1}$ we usually have $\alpha_{X}^{*} \neq \beta_{X}$. Theorem 2.3 describes the Hilbert function $H_{X}(i, j)$ for $(i, j) \gg(0,0)$ in terms of the partitions $\alpha, \beta$. It was first formulated by Van Tuyl [16], here we use the statement from Guardo and Van Tuyl [12].

Theorem 2.3 (Guardo-Van Tuyl [12]). Let $X \subset \mathbb{P}^{1} \times \mathbb{P}^{1}$ be any set of points with associated tuples $\alpha_{X}=\left(\alpha_{1}, \ldots, \alpha_{h}\right)$ and $\beta_{X}=\left(\beta_{1}, \ldots, \beta_{\nu}\right)$ and let $h=\left|\pi_{1}(X)\right|$ and $\nu=\left|\pi_{2}(X)\right|$.

(1) For all $j \in \mathbb{N}$, if $i \geq h-1$, then

$$
H_{X}(i, j)=\alpha_{1}^{*}+\cdots+\alpha_{j+1}^{*}
$$

where $\alpha_{X}^{*}=\left(\alpha_{1}^{*}, \ldots, \alpha_{\alpha_{1}}^{*}\right)$ is the conjugate of $\alpha_{X}$ and where we make the convention that $\alpha_{l}^{*}=0$ if $l>\alpha_{1}$.

(2) For all $i \in \mathbb{N}$, if $j \geq \nu-1$, then

$$
H_{X}(i, j)=\beta_{1}^{*}+\cdots+\beta_{j+1}^{*}
$$

where $\beta_{X}^{*}=\left(\beta_{1}^{*}, \ldots, \beta_{\beta_{1}}^{*}\right)$ is the conjugate of $\beta_{X}$ and where we make the convention that $\beta_{l}^{*}=0$ if $l>\beta_{1}$.

Thus if we know the values of $H_{X}(i, j)$ for $(i, j) \gg 0$ we are able to determine $\alpha$ and $\beta$. This in turn gives us information about the vertical an horizontal rulings that contain $X$. We will use this theorem to give a geometric description of a generic set of $r$ points in $\mathbb{P}^{1} \times \mathbb{P}^{1}$ in subsection 3.2 .

Example 2.4. Let $X$ be the set of points in $\mathbb{P}^{1} \times \mathbb{P}^{1}$ on the left below. Then $\alpha_{X}=$ $(4,4,3,2), \beta_{X}=(3,2,2,2,2,2)$ and $\alpha_{X}^{*}=(4,4,3,2), \beta_{X}^{*}=(6,6,1)$. The previous theorem implies that $H_{X}$ has the following form.

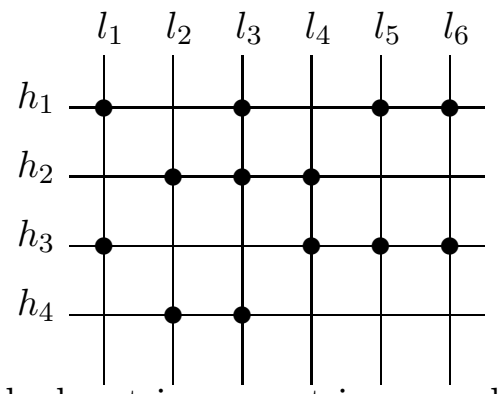

$H_{X}=$\begin{tabular}{c||c|c|c|c|c|c|c} 
& 0 & 1 & 2 & 3 & 4 & 5 & 6 \\
\hline \hline 0 & & & & & & 6 & 6 \\
\hline 1 & & & & & & 12 & 12 \\
\hline 2 & & & & & & 13 & 13 \\
\hline 3 & 4 & 8 & 11 & 13 & 13 & 13 & 13 \\
\hline 4 & 4 & 8 & 11 & 13 & 13 & 13 & 13
\end{tabular}

The blank entries cannot in general be deduced from the information contained in $\alpha_{X}$ and $\beta_{X}$. In Examples 3.4 and 3.5 we illustrate this fact with two sets of points $X_{1}, X_{2}$ in $\mathbb{P}^{1} \times \mathbb{P}^{1}$ whose Hilbert function is different but for which $\alpha_{X_{1}}=\alpha_{X_{2}}$ and $\beta_{X_{1}}=\beta_{X_{2}}$. 


\section{IDEAL OF A GENERIC SET OF POINTS IN $\mathbb{P}^{1} \times \mathbb{P}^{1}$}

In this section we describe minimal generators of $I_{X}$ in bidegree $(a, 1)$ when $X$ is a generic set of points of $\mathbb{P}^{1} \times \mathbb{P}^{1}$. For this purpose we use the work of Van Tuyl [17 for the case of $\mathbb{P}^{1} \times \mathbb{P}^{1}$. Using this description in Section 4 will allow us to find syzygies of $I_{U}=\left\langle f_{0}, \ldots, f_{3}\right\rangle$.

\subsection{Description of $I_{X}$.}

Definition 3.1. Let $X$ be a set of $r$ points in $\mathbb{P}^{1} \times \mathbb{P}^{1}$. The set $X$ is said to be generic if its bigraded Hilbert function, is determined by

$$
H_{X}(i, j)=\min \{(i+1)(j+1), r\} .
$$

Being a generic set of points is a property of the Hilbert function. Note that from the definition of a generic set of points in $\mathbb{P}^{1} \times \mathbb{P}^{1}$ we cannot immediately describe the bidegrees of the minimal generators of $I_{X}$ nor the distribution of the points of $X$ in families of $(1,0)$ and $(0,1)$ lines. We focus on describing these two aspects for the rest of this section.

If $\mathcal{E}$ is any subset of $\mathbb{N}^{2}$ and $\underline{a}=\left(a_{0}, a_{1}\right) \in \mathbb{N}^{2}$ is any tuple, then $\mathcal{E}+\underline{a}$ denotes the set $\{\underline{e}+\underline{a}: \underline{e} \in \mathcal{E}\}$. Let $e_{1}=(1,0)$ and $e_{2}=(0,1)$.

For every $i \geq 0$

$$
j(i):=\min \left\{t \in \mathbb{N} \mid H_{X}(i, t)=H_{X}(i, t+1)\right\}
$$

and for every $j \geq 0$

$$
i(j):=\min \left\{t \in \mathbb{N} \mid H_{X}(t, j)=H_{X}(t+1, j)\right\} .
$$

Theorem 3.2 (Van Tuyl[17]). Let $X$ be a finite set of points in $\mathbb{P}^{1} \times \mathbb{P}^{1}$. Fix $e_{1}=(1,0)$ and $j \in \mathbb{N}$. Set $\underline{i}=(i(j), j)$. Then

$$
\left(I_{X}\right)_{\underline{i}+(q+1) e_{1}}=R_{e_{1}}\left(I_{X}\right)_{\underline{i}+q e_{1}} \quad \forall q \in \mathbb{N} .
$$

In particular, if there exists $\underline{l} \in \mathbb{N}^{2}$ and $t \in\{1,2\}$ such that $H_{X}(\underline{l})=H_{X}\left(\underline{l}-e_{t}\right)=$ $H_{X}\left(\underline{l}-2 e_{t}\right)$. Then $I_{X}$ has no minimal generators of bidegree $\underline{l}$.

Theorem 3.2 gives a way to exclude bidegrees that will not show up as bidegrees of minimal generators for $I_{X}$. It also gives us a description of the higher degree pieces of $I_{X}$ once the bigraded Hilbert function has stabilized in one of the coordinates.

Define the $k[s, t]$-module $M$ by

$$
M=\bigoplus_{i=0}^{\infty}\left(I_{X}\right)_{(i, 1)} .
$$


Following the notation of Theorem 3.2, fix $j=1$, then $i(1)$ is the value of $i$ at which the bigraded Hilbert function $H_{X}$ of $X$ stabilizes for the column $j=1$. So for all $q \in \mathbb{N}$,

$$
H_{X}(i(1), 1)=H_{X}(i(1)+q, 1)
$$

Recall that $r$ is the number of points in $X$, we want to find $i(1)$ and understand the generators of $M$ as a $k[s, t]$ module.

Proposition 3.3. Let $X$ be a generic set of $r$ points in $\mathbb{P}^{1} \times \mathbb{P}^{1}$ with associated bihomogeneous ideal $I_{X}$. Then the $k[s, t]$-module $M$ has two minimal generators in bidegrees $(k, 1),(k, 1)$ if $r=2 k$ and two minimal generators in bidegrees $(k, 1),(k+1,1)$ if $r=2 k+1$.

Proof. Suppose $r=2 k$. Then

$$
H_{X}(i, 1)=\min \{r,(i+1) 2\} .
$$

For all $0 \leq i<k-1, H_{X}(i, 1)=(i+1) 2$ and for $i \geq k-1, H_{X}(i, 1)=r$. Thus $i(1)=k-1$ and we have

$$
r=H_{X}(k-1,1)=H_{X}(k, 1)=H_{X}(k+1,1) .
$$

Using Theorem 3.2 we know that $I_{X}$ has no minimal generators of degree $(k+1,1)$ or higher. Thus possible minimal generators of $I_{X}$ are in bidegrees $(k-1,1),(k, 1)$. But we have

$$
H_{X}(k-1,1)=r=\operatorname{dim} R_{(k-1,1)}-\operatorname{dim}\left(I_{X}\right)_{(k-1,1)},
$$

so $\operatorname{dim}\left(I_{X}\right)_{(k-1,1)}=0$ and $I_{X}$ does not have generators in bidegree $(k-1,1)$. A similar equation to (1) shows that $\operatorname{dim}\left(I_{X}\right)_{(k, 1)}=2$. Therefore $M$ has two generators of bidegree $(k, 1)$. Moreover, using the last part of Theorem 3.2 we have

$$
\left(I_{X}\right)_{i(1)+(q+1) e_{1}}=R_{e_{1}}\left(I_{X}\right)_{i(1)+q e_{1}} \quad \forall q \in \mathbb{N} .
$$

Since $R_{e_{1}}=\{s, t\}$ this shows $M$ has two generators in bidegree $(k, 1)$ as a $k[s, t]$ module. The proof for the case when $r$ is odd is done in a similar way.

3.2. Geometric description of $X$ in terms of rulings. Let $X$ be a set of generic points in $\mathbb{P}^{1} \times \mathbb{P}^{1}$. Using the Hilbert function of $X$, we see that for $(i, j)$ with $0 \leq j$ and $i \geq r-1$, $H_{X}(i, j)=r$ and for $(i, j)$ with $0 \leq i$ and $j \geq r-1, H_{X}(i, j)=r$. Using Theorem 2.3, we know that for $j=0$ and $i \gg 0, H_{X}(i, 0)=\alpha_{1}^{*}=r$. Since $X$ consists exactly of $r$ points, we conclude that $\alpha_{i}^{*}=0$ for all $i>0$ and $\alpha_{X}=(1, \ldots, 1)$ has $r$ parts. Similarly, $\beta_{X}=(1, \ldots, 1)$ has $r$ parts. Using the definition of $\alpha_{X}$, we know that the number of parts of $\alpha_{X}$ is equal to the number of horizontal lines in $\mathbb{P}^{1} \times \mathbb{P}^{1}$ that contain points of $X$. 
Moreover, each part $\alpha_{i}$ of $\alpha_{X}$ corresponds to the number of points in $X$ that lie on the horizontal line $h_{i}$. From $\alpha_{X}=(1, \ldots, 1)$, we conclude that there are $r$ distinct horizontal lines in $\mathbb{P}^{1} \times \mathbb{P}^{1}$ each containing exactly one point of $X$. We have a similar statement for $\beta_{X}$ and vertical lines. After reordering of the vertical and horizontal lines that contain points of $X$, we can describe $X$ as the set of diagonal points inside an $r \times r$ grid of lines.

Example 3.4. Let $I_{X_{1}}=(s, u) \cap(t, v) \cap(s-3 t, u-v) \cap(s+t, u+5 v)$. Using Macaulay2 we compute the bigraded Hilbert function $H_{X_{1}}$ on the right and conclude $X_{1}$ is generic. The distribution of $X_{1}$ on the rulings of $\mathbb{P}^{1} \times \mathbb{P}^{1}$ is illustrated on the left.

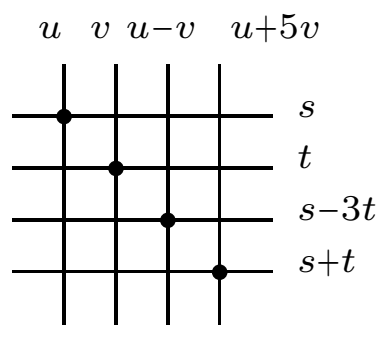

$$
H_{X}=\begin{array}{c||c|c|c|c|c} 
& 0 & 1 & 2 & 3 & 4 \\
\hline \hline 0 & 1 & 2 & 3 & 4 & 4 \\
\hline 1 & 2 & 4 & 4 & 4 & 4 \\
\hline 2 & 3 & 4 & 4 & 4 & 4 \\
\hline 3 & 4 & 4 & 4 & 4 & 4 \\
\hline 4 & 4 & 4 & 4 & 4 & 4
\end{array}
$$

Using Proposition 3.3, we know $I_{X_{1}}$ has two generators $g_{1}, g_{2}$ in bidegree $(2,1)$. A Macaulay 2 computation yields $g_{1}=6 t^{2} u+7 s^{2} v-23 s t v, g_{2}=2 s t u-3 s^{2} v+7 s t v$.

Example 3.5. Let $I_{X_{2}}=(s, u) \cap(t, v) \cap(s-t, u-v) \cap(s+t, u+v)$. Using Macaulay2 we compute the bigraded Hilbert function $H_{X_{2}}$ on the right and conclude that $X_{2}$ is not generic.

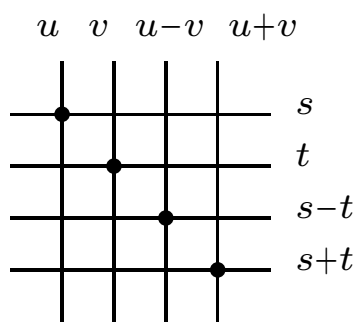

$H_{X}=$\begin{tabular}{c||c|c|c|c|c} 
& 0 & 1 & 2 & 3 & 4 \\
\hline \hline 0 & 1 & 2 & 3 & 4 & 4 \\
\hline 1 & 2 & 3 & 4 & 4 & 4 \\
\hline 2 & 3 & 4 & 4 & 4 & 4 \\
\hline 3 & 4 & 4 & 4 & 4 & 4 \\
\hline 4 & 4 & 4 & 4 & 4 & 4
\end{tabular}

The ideal $I_{X_{2}}$ has a minimal generator of bidegree $(1,1)$ whereas the generic set of points $X_{1}$ from Example 3.4 has no generator of such bidegreee. However we have $\alpha_{X_{1}}=\alpha_{X_{2}}$ and $\beta_{X_{1}}=\beta_{X_{2}}$. In this case the module $M$ from Proposition 3.3 has two generators of bidegrees $(1,1)$ and $(3,1)$. 


\section{Syzygies of the IDEAL $I_{U}$}

As was highlighted in the introduction, the syzygies of $I_{U}$ determine the complex $\mathcal{Z}$ which in turn determine the implicit equation of $X_{U}$. Let $\operatorname{Syz}\left(I_{U}\right)_{(-, 0)}$ denote the $k[s, t]$-module of syzygies of $I_{U}=\left\langle f_{0}, \ldots, f_{3}\right\rangle \subset R$ of degree zero in $u, v$. The following proposition states that $\operatorname{Syz}\left(I_{U}\right)_{(-, 0)}$ has rank two and gives the bidegrees of its minimal generators.

Proposition 4.1. Let $X$ be a generic set of $r$ points in $\mathbb{P}^{1} \times \mathbb{P}^{1}$ and let $U=\left\{f_{0}, \ldots, f_{3}\right\}$ be a generic 4-dimensional vector space of $\left(I_{X}\right)_{(a, 1)}$ with $a \geq k$. Then the $k[s, t]$-module $\operatorname{Syz}\left(I_{U}\right)_{(-, 0)}$ has rank two. Moreover, $\operatorname{Syz}\left(I_{U}\right)_{(-, 0)}$ has minimal generators of bidegree $(a-k, 0)$ if $r=2 k$ and of bidegrees $(a-k, 0),(a-k+1,0)$ if $r=2 k+1$.

Remark 4.2. If $\mathbf{b}=\left\{b_{1}, \ldots, b_{q}\right\}$ is a basis for $\left(I_{X}\right)_{(a, 1)}$, we make the convention that a generic choice of $U$ is given by a matrix $C$ of size $4 \times q$ with $\mathbf{f}=C \mathbf{b}$ all of whose maximal minors are nonzero.

The proof of Proposition 4.1 will be done in four steps. The steps that will follow are done for the case $r=2 k$. If $r=2 k+1$, similar steps work by changing to $(a-k, 0)$ and $(a-k+1,0)$.

Step 1. Use the description of a basis for $\left(I_{X}\right)_{(a, 1)}$ to show that the generators of the ideal $I_{U}$ may be written in a simpler and more convenient form.

From Proposition 3.3 we know $\left(I_{X}\right)_{(k, 1)}$ is generated by two elements $g_{1}, g_{2}$. Thus by equation (2) in the proof of Proposition 3.3, a basis for $\left(I_{X}\right)_{(a, 1)}$, is given by

$$
\mathbf{b}=\left\{s^{j} g_{1}, s^{j-1} t g_{1}, \ldots, t^{j} g_{1}, s^{j} g_{2}, \ldots, s t^{j-1} g_{2}, t^{j} g_{2}\right\},
$$

where $j=a-k$. Following our convention from Remark 4.2, a generic choice of $U$ is a coefficient matrix $C$ such that every maximal minor of $C$ is nonzero with $\mathbf{f}=C \mathbf{b}$. Using the basis $\mathbf{b}$ for $\left(I_{X}\right)_{(a, 1)}$

$$
\left(\begin{array}{llll}
f_{0} & f_{1} & f_{2} & f_{3}
\end{array}\right)^{T}=\left(\begin{array}{lllllll}
s^{j} g_{1} & s^{j-1} t g_{1} & \cdots & t^{j} g_{1} & s^{j} g_{2} & \cdots & t^{j} g_{2}
\end{array}\right) C^{T} .
$$

The first $j+1$ elements of the basis share the common factor $g_{1}$ and the last $j+1$ elements share the factor $g_{2}$, thus we may write

$$
\left(\begin{array}{llll}
f_{0} & f_{1} & f_{2} & f_{3}
\end{array}\right)=\left(\begin{array}{ll}
g_{1} & g_{2}
\end{array}\right)\left(\begin{array}{cccc}
Q_{0} & Q_{1} & Q_{2} & Q_{3} \\
P_{0} & P_{1} & P_{2} & P_{3}
\end{array}\right)
$$

where $\operatorname{deg} Q_{i}=\operatorname{deg} P_{i}=(j, 0)$ are forms in the variables $s, t$. Denote the rightmost matrix in (3) by $Q P$. 
Using the notation in the first step we let

$$
\left(\begin{array}{llll}
Q_{0} & Q_{1} & Q_{2} & Q_{3}
\end{array}\right)=\left(\begin{array}{lll}
s^{j} & s^{j-1} t \cdots t^{j}
\end{array}\right) A^{T},
$$

and

$$
\left(\begin{array}{llll}
P_{0} & P_{1} & P_{2} & P_{3}
\end{array}\right)=\left(\begin{array}{lll}
s^{j} & s^{j-1} t \cdots t^{j}
\end{array}\right) B^{T} .
$$

Denote the matrix of coefficients of $Q_{i}$ 's by $A$ and the matrix of coefficients of $P_{i}$ 's by $B$. Observe that $\mathrm{C}$ is the block matrix formed by $A$ and $B$, so $C=(A \mid B)$.

Step 2. Reduce the problem to the study of the kernel of the matrix $Q P$.

Lemma 4.3. The ideal $I_{U}$ has a syzygy $L$ of bidegree $(\alpha, 0)$ if and only if $L$ is an element in the kernel of the matrix

$$
Q P=\left(\begin{array}{cccc}
Q_{0} & Q_{1} & Q_{2} & Q_{3} \\
P_{0} & P_{1} & P_{2} & P_{3}
\end{array}\right)
$$

over the ring $k[s, t]$.

Proof. Suppose that $L=\left(s_{0}, s_{1}, s_{2}, s_{3}\right)$ is a syzygy of $\left(f_{0}, f_{1}, f_{2}, f_{3}\right)$ of degreee $(\alpha, 0)$. Then

$$
s_{0} f_{0}+s_{1} f_{1}+s_{2} f_{2}+s_{3} f_{3}=0 .
$$

Using Step 1, $f_{i}=Q_{i} g_{1}+P_{i} g_{2}$. We may substitute this in the previous equation and factor $g_{1}$ and $g_{2}$ as follows,

$$
\left(s_{0} Q_{0}+s_{1} Q_{1}+s_{2} Q_{2}+s_{3} Q_{3}\right) \cdot g_{1}+\left(s_{0} P_{0}+s_{1} P_{1}+s_{2} P_{2}+s_{3} P_{3}\right) \cdot g_{2}=0
$$

The elements $g_{1}, g_{2}$ are minimal generators of $I_{X}$ and thus form a complete intersection. Using the fact that the only syzygies of a complete intersection are Koszul we obtain

$$
\left(\begin{array}{c}
s_{0} Q_{0}+s_{1} Q_{1}+s_{2} Q_{2}+s_{3} Q_{3} \\
s_{0} P_{0}+s_{1} P_{1}+s_{2} P_{2}+s_{3} P_{3}
\end{array}\right)=q\left(\begin{array}{r}
-g_{2} \\
g_{1}
\end{array}\right) .
$$

Note that $\operatorname{deg} s_{0} Q_{0}+s_{1} Q_{1}+s_{2} Q_{2}+s_{3} Q_{3}=\operatorname{deg} s_{0} P_{0}+s_{1} P_{1}+s_{2} P_{2}+s_{3} P_{3}=(\alpha+j, 0)$. But $\operatorname{deg} g_{i}=(k, 1)$, hence $q=0$ and

$$
\left(\begin{array}{c}
s_{0} Q_{0}+s_{1} Q_{1}+s_{2} Q_{2}+s_{3} Q_{3} \\
s_{0} P_{0}+s_{1} P_{1}+s_{2} P_{2}+s_{3} P_{3}
\end{array}\right)=0
$$

so $L \in \operatorname{ker} Q P$. Verifying the other direction is straightforward from the previous calculations. 
Step 3. When $Q P$ is a matrix whose entries are polynomials in $k[s, t]$ of degree $j$, with coefficients from a generic matrix $C$ as in equations (4) and (5), the kernel of $Q P$ has rank two and its minimal generators are of degree $j$.

A quick check reveals that the assumption $\operatorname{gcd}\left(f_{0}, \ldots, f_{3}\right)=1$ implies the matrix $Q P$ has rank two. Then it can be proved that if the coefficients of $Q P$ are chosen generically following Remark 4.2, the two minimal generators of ker $Q P$ have degree $j=a-k$. If the coefficients of $Q P$ are not chosen generically, we can expect the degrees of the two minimal generators of $\operatorname{ker} Q P$ to be less than or equal to $j$.

Step 4. Using Step 2, we know that the syzygies of $I_{U}$ in bidegree $(\alpha, 0)$ are in one-to-one correspondence with elements in the kernel of $Q P$. Following Step 3 , the kernel of $Q P$ is generated by two elements $K 1, K 2$ of degree $j$ in $s, t$, hence $I_{U}$ has two minimal syzygies of bidegree $(j, 0)$.

Remark 4.4. Note that the two elements $K 1, K 2$ in the kernel of $Q P$ generate a free module. Indeed, $Q P$ fits into a sequence

$$
0 \longrightarrow S^{2} \longrightarrow S^{4} \stackrel{Q P}{\longrightarrow} S^{2} \longrightarrow \operatorname{coker} Q P \longrightarrow 0
$$

where the leftmost nonzero map sends $S^{2}$ to the two syzygies $K 1, K 2$ and $S=k[s, t]$. Then by Hilbert's syzygy theorem over $S$, the leftmost map in the sequence (6) is injective.

\section{Applications to implicitization of tensor PRODUCT SURFACES}

We now focus on obtaining the implicit equation of $X_{U}$, when $U=\left\{f_{0}, \ldots, f_{3}\right\} \subseteq$ $\left(I_{X}\right)_{(a, 1)}$ is a generic 4-dimensional subspace. First we give a definition of $\mathcal{Z}$ and explain how to set up the complex $\mathcal{Z}_{\nu}$ with an example. Next, we give a proof of the main theorem and apply these techniques for the basepoint free case. We also illustrate the advantages of the syzygy method over Gröbner bases. All of the examples presented in this section were computed using the software Macaulay2. Throughout this section, the implicit equation of a parameterized surface in $\mathbb{P}^{3}$ will be denoted by $H$ and it is an element in $S=k[X, Y, Z, W]$.

5.1. Implicitization via syzygies. Given $U=\left\{f_{0}, \ldots, f_{3}\right\}$, the complex $\mathcal{Z}$ has $\mathcal{Z}_{i}=$ $\operatorname{ker}\left(d_{i}^{f}\right) \otimes S$ where $d_{i}^{f}$ is the differential of the Koszul complex associated with the sequence $f=\left(f_{0}, \ldots, f_{3}\right)$ and $S=k[X, Y, Z, W]$ is the coordinate ring of $\mathbb{P}^{3}$. The differential $\partial_{i}: \mathcal{Z}_{i} \rightarrow \mathcal{Z}_{i-1}$ for the complex $\mathcal{Z}$ is defined to be the differential of the Koszul complex associated with the sequence $(X, Y, Z, W)$. We use the results by Botbol [1] 
to compute the implicit equation of $X_{U}$ via the approximation complex $\mathcal{Z}$. Recall that $\mathcal{Z}_{1}=\operatorname{Syz}\left(f_{0}, \ldots, f_{3}\right) \otimes S$.

Theorem 5.1 (Botbol [1]). Let $\lambda: \mathbb{P}^{1} \times \mathbb{P}^{1} \rightarrow-\mathbb{P}^{3}$ be a rational map with finitely many local complete intersection basepoints given by 4 bihomogeneous polynomials $f_{0}, \ldots, f_{3} \in R_{(a, b)}$ without common factors. Then for $\nu=(2 a-1, b-1)$ (equiv. $\nu=(a-1,2 b-1))$

$$
\operatorname{det} \mathcal{Z}_{\nu}=H^{\operatorname{deg} \lambda} \in k[X, Y, Z, W],
$$

where $H$ is the irreducible implicit equation of $X_{U}$.

Since $U \subseteq\left(I_{X}\right)_{(a, 1)}, X$ is the set of basepoints of $X_{U}$ and we are able to use Theorem 5.1 . To illustrate how the computation of the implicit equation works we have the following example.

Example 5.2. Let $X$ be the set of points in $\mathbb{P}^{1} \times \mathbb{P}^{1}$ associated to the ideal $I_{X}=(s, u) \cap$ $(t, v)=(u v, s v, t u, s t)$. Computing the Hilbert function of $X$ in Macaulay2 shows that $X$ is generic. Take $(a, 1)=(3,1)$, then

$$
\left(I_{X}\right)_{(3,1)}=\left\{s^{3} v, t^{3} u, s^{2} t u, s^{2} t v, s t^{2} u, s t^{2} v\right\} .
$$

We take $U$ to be the generic 4 -dimensional subspace given by

$$
U=\left\{s^{3} v-s t^{2} u+s t^{2} v, t^{3} u+s t^{2} u+s t^{2} v, s^{2} t u+s t^{2} u-3 s t^{2} v, s^{2} t v-5 s t^{2} u+s t^{2} v\right\} .
$$

To compute the implicit equation of $X_{U}$, we need to set up the complex $\mathcal{Z}_{\nu}$ and then find $\operatorname{det} \mathcal{Z}_{\nu}$. The complex $\mathcal{Z}_{\nu}$,

$$
\mathcal{Z}_{\nu}: \quad 0 \longrightarrow\left(\mathcal{Z}_{2}\right)_{\nu} \stackrel{d_{2}}{\longrightarrow}\left(\mathcal{Z}_{1}\right)_{\nu} \stackrel{d_{1}}{\longrightarrow}\left(\mathcal{Z}_{0}\right)_{\nu} \longrightarrow 0
$$

only has three nonzero terms and it is exact. Following Theorem 5.1, $\nu=(5,0)$. The ideal $I_{U}$ has two syzygies in degree $(2,0)$ and three syzygies in bidegree $(1,1)$. The syzygies in degree $(2,0)$ span a free $R$-module and determine a basis for the syzygies of bidegree $(5,0)$ by multiplying by $\left\{s^{3}, s^{2} t, s t^{2}, t^{3}\right\}$. We proceed to apply the map $\partial_{1}$ to this basis and obtain the matrix $d_{1}$. Using Macaulay 2 and $d_{2}=$ ker $d_{1}$ we obtain

$d_{1}=\left(\begin{array}{lllllll}107 Y & 0 & 0 & 0 & 0 & 107 W & 0 \\ -228 Y-107 Z-52 W & 107 Y & 0 & 0 & -107 X+1082 Y+535 Z+335 W & 107 W & 0 \\ -55 X-32 Z-41 W & -228 Y-107 Z-52 W & 107 Y & 0 & -442 X-49 Z+295 W & -107 X+1082 Y+535 Z+335 W & 107 W \\ 0 & -55 X-32 Z-41 W & -228 Y-107 Z-52 W & 107 Y & 0 & -442 X-49 Z+295 W & -107 X+1082 Y+535 Z+335 W \\ 0 & 0 & -55 X-32 Z-41 W & -228 Y-107 Z-52 W & 0 & 0 & -407 W \\ 0 & 0 & 0 & -55 X-32 Z-41 W & 0 & 0 & -107 X+1082 Y+535 Z+335 W\end{array}\right)$,




$$
d_{2}=\left(\begin{array}{ll}
-1 / 107 W & 0 \\
1 / 107 X-1082 / 11449 Y-5 / 107 Z-335 / 11449 W & -1 / 107 W \\
442 / 11449 X+49 / 11449 Z-295 / 11449 W & 1 / 107 X-1082 / 11449 Y-5 / 107 Z-335 / 11449 W \\
0 & 442 / 11449 X+49 / 11449 Z-295 / 11449 W \\
1 / 107 Y & 0 \\
-228 / 11449 Y-1 / 107 Z-52 / 11449 W & 1 / 107 Y \\
-55 / 11449 X-32 / 11449 Z-41 / 11449 W & -228 / 11449 Y-1 / 107 Z-52 / 11449 W \\
0 & -55 / 11449 X-32 / 11449 Z-41 / 11449 W
\end{array}\right) .
$$

The determinant of $\mathcal{Z}_{\nu}$ is computed by $\operatorname{det} \mathcal{Z}_{\nu}=\operatorname{det} M_{1} / \operatorname{det} M_{2}$, where $M_{1}$ is a maximal nonzero minor of $d_{1}$ and $M_{2}$ is the complimentary maximal nonzero minor of $d_{2}$,

$$
\begin{aligned}
& -8831798120631365 X^{3} Y+623043212873630840 X^{2} Y^{2}-2432437780569525764 X Y^{3} \\
& +154155021741929280 X^{2} Y Z-2181293557648299312 X Y^{2} Z-694982223019864504 Y^{3} Z- \\
& 516419322835463088 X Y Z^{2}-679406142698023733 Y^{2} Z^{2}-167804164291995935 Y Z^{3} \\
& +29064644724259583 X^{2} Y W-2253232567794532976 X Y^{2} W+347491111509932252 Y^{3} W+ \\
\operatorname{det} \mathcal{Z}_{\nu}= & 8831798120631365 X^{2} Z W-1142192364219107259 X Y Z W-288398353175526028 Y^{2} Z W- \\
& 109996031138772455 X Z^{2} W-277318460987824861 Y Z^{2} W-33560832858399187 Z^{3} W \\
& +8831798120631365 X^{2} W^{2}-417342605736743957 X Y W^{2}+335768906731639713 Y^{2} W^{2} \\
& -103733483380506578 X Z W^{2}+6583704053561563 Y Z W^{2}-20232846603628218 Z^{2} W^{2}- \\
& 20232846603628218 X W^{3}+65676462387967787 Y W^{3}+3693297395900389 Z W^{3}+ \\
& 3693297395900389 W^{4} .
\end{aligned}
$$

As was mentioned in the introduction, the matrix $d_{1}$ representing the map $\left(\mathcal{Z}_{\nu}\right)_{1} \rightarrow\left(\mathcal{Z}_{\nu}\right)_{0}$ is sufficient to perform the elementary geometric operations that arise in CAGD. For this reason the growth of the size of the coefficients for the implicit equation in practice does not affect the speed of the computations.

5.2. Proof of main theorem. To prove Theorem 1.1 we need two lemmas, the first one states the dimension of $\left(\mathcal{Z}_{1}\right)_{\nu}$ and the second one provides a basis for $\left(\mathcal{Z}_{1}\right)_{\nu}$.

Lemma 5.3. Let $\mathcal{Z}$ be the approximation complex associated to $\left(f_{0}, \ldots, f_{3}\right)$, with differential on the variables $(X, Y, Z, W)$. If $U=\left\{f_{0}, \ldots, f_{3}\right\}$ has $r$ basepoints, each of multiplicity one, then

$$
\operatorname{dim}_{k}\left(\mathcal{Z}_{1}\right)_{\nu}=2 a b+r
$$

where $\nu=(2 a-1, b-1)$.

Proof. From the proof of Lemma 7.3 in Botbol [1, we know that $\mathcal{Z}_{\nu}$ is acyclic and that $\left(\mathcal{Z}_{3}\right)_{\nu}=0$. Hence $\mathcal{Z}_{\nu}$ has the form

$$
\mathcal{Z}_{\nu}: \quad 0 \longrightarrow\left(\mathcal{Z}_{2}\right)_{\nu} \longrightarrow\left(\mathcal{Z}_{1}\right)_{\nu} \longrightarrow\left(\mathcal{Z}_{0}\right)_{\nu} \longrightarrow 0 .
$$

Using Lemma 7.3 and 7.4 from Botbol [1, and the fact that $I_{U}$ is a local complete intersection, Botbol observes that

$$
\operatorname{dim}_{k}\left(\mathcal{Z}_{2}\right)_{\nu}=\sum_{x \in X} e_{x}
$$


where $e_{x}$ denotes the multiplicity of the basepoint $x$ of $U$ and $X$ is the set of basepoints of $U$. Since $U$ has $r$ basepoints each of multiplicity one, $\operatorname{dim}_{k}\left(\mathcal{Z}_{2}\right)_{\nu}=r$. Also, $\left(\mathcal{Z}_{0}\right)_{\nu}=$ $R_{(2 a-1, b-1)}$, thus $\operatorname{dim}_{k}\left(\mathcal{Z}_{0}\right)_{\nu}=2 a b$. It follows that $\operatorname{dim}_{k}\left(\mathcal{Z}_{1}\right)_{\nu}=2 a b+r$.

Lemma 5.4. Let $U=\left\{f_{0}, \ldots, f_{3}\right\}$ be a generic 4-dimensional vector subspace of $\left(I_{X}\right)_{(a, 1)}$ and $\mathcal{Z}$ the approximation complex associated to $\left(f_{0}, \ldots, f_{3}\right)$. Then a basis for $\left(\mathcal{Z}_{1}\right)_{\nu}$, $\nu=(2 a-1, b-1)$, is obtained by bumping up the syzygies of $I_{U}$ in bidegree $(a-k, 0)$ if $r=2 k$ and the syzygies in bidegrees $(a-k, 0),(a-k+1,0)$ if $r=2 k+1$.

Proof. We have $\mathcal{Z}_{1}=\operatorname{ker}\left(d_{1}^{f}\right)=\operatorname{Syz}\left(f_{0}, \ldots, f_{3}\right)$, thus $\left(\mathcal{Z}_{1}\right)_{\nu}=\left(\operatorname{Syz}\left(f_{0}, \ldots, f_{3}\right)\right)_{\nu}$. If $r$ is even then $I_{U}$ has two syzygies $S_{1}, S_{2}$ of bidegree $(a-k, 0)$ by Proposition 4.1. Using Remark 4.4 at the end of Section 4 , we know that $S_{1}, S_{2}$ span a free module. Hence the set of syzygies $\mathcal{S}=\left\{m \cdot S_{i} \mid m \in R_{(a+k-1,0)}, i=1,2\right\}$ is an independent subset of $\left(\operatorname{Syz}\left(f_{0}, \ldots, f_{3}\right)\right)_{\nu}$. Note that $\operatorname{dim}_{k} R_{(a+k-1,0)}=a+k$, thus $\operatorname{dim}_{k} \operatorname{Span} \mathcal{S}=2 a+r$. From Lemma $5.3 \operatorname{dim}\left(\mathcal{Z}_{1}\right)_{\nu}=2 a+r$, hence a basis for $\left(\mathcal{Z}_{1}\right)_{\nu}$ is determined by bumping up $S_{1}, S_{2}$. An analogous argument works in the case $r$ is odd.

We are now ready to prove Theorem 1.1

Theorem. Let $\left(I_{X}\right) \subseteq H^{0}(a, 1)$ be the $k$-vector space of forms of bidegree $(a, 1)$ that vanish at a generic set $X$ of $r$ points in $\mathbb{P}^{1} \times \mathbb{P}^{1}$. Take $U=\left\{f_{0}, \ldots, f_{3}\right\}$ to be a generic 4-dimensional vector subspace of $\left(I_{X}\right)_{(a, 1)}$ and $\lambda_{U}: \mathbb{P}^{1} \times \mathbb{P}^{1} \rightarrow \mathbb{P}^{3}$ the rational map determined by $U$. Then the first map of the approximation complex $\mathcal{Z}$ in bidegree $\nu=(2 a-1,0)$ is determined by the syzygies of $\left(f_{0}, \ldots, f_{3}\right)$ in bidegree $(a-k, 0)$ if $r=2 k$ and bidegrees $(a-k, 0),(a-k+1,0)$ if $r=2 k+1$. These syzygies completely determine the complex $\mathcal{Z}_{\nu}$ from which we compute the implicit equation.

Proof. We consider the case $r=2 k$, the case $r=2 k+1$ is done similarly. Let $S_{1}, S_{2}$ be the two minimal syzygies of $I_{U}$ in bidegree $(a-k, 0)$. By Lemma 5.4, $\left(\mathcal{Z}_{1}\right)_{\nu}$ has a basis given by the elements of the form $m \cdot S_{i}$ where $i=1,2$ and $m \in R_{(a+k-1,0)}$. Denote the matrix of the first map of the complex $\mathcal{Z}_{\nu}$ by $d_{1}$. Then $d_{1}$ is obtained by applying the Koszul differential on the sequence $(X, Y, Z, W)$ to all the elements $\left\{m \cdot S_{i}\right\}$. Using the proof of Lemma 5.3 , we know $\mathcal{Z}_{\nu}$ is exact, hence $d_{2}=$ ker $d_{1}$. Therefore the approximation complex $\mathcal{Z}_{\nu}$ that determines the implicit equation of $X_{U}$ only depends on the syzygies of $I_{U}$ in degree $(j, 0)$.

Corollary 5.5. Let $X$ be a set of $r$ points in $\mathbb{P}^{1} \times \mathbb{P}^{1}$ with $r=2 k$. Take $U$ to be any 4-dimensional vector subspace of $\left(I_{X}\right)_{(k+1,1)}$, then $X_{U}$ is smooth. 
Proof. From Proposition 3.3. $\left(I_{X}\right)_{(k+1,1)}=\left\{s g_{1}, t g_{1}, s g_{2}, t g_{2}\right\}$ so $\operatorname{dim}_{k}\left(I_{X}\right)_{(k+1,1)}=4$. Thus up to a change of coordinates, any choice of $U$ is equivalent to the choice $U=$ $\left\{s g_{1}, t g_{1}, s g_{2}, t g_{2}\right\}$. For this $U$, it follows that $X_{U}=\mathbb{V}(X W-Y Z)$, hence $X_{U}$ is smooth.

The results in Theorem 1.1 do not generalize immediately to tensor product surfaces of more general bidegree $(a, b)$ with $b>1$. One of the advantages of the condition $b=1$ is that the calculation of the syzygies of $I_{U}$ is reduced to finding the kernel of the matrix $Q P$ over the polynomial ring $k[s, t]$ which has two fewer variables than $R$. This allows us to show in Remark 4.4 that the syzygies of $I_{U}$ are free. For more general bidegree, the syzygies of $I_{U}$ are not free and computing a basis for $\operatorname{Syz}\left(I_{U}\right)_{\nu}$ is more difficult because of the possible relations between the generators of $\operatorname{Syz}\left(I_{U}\right)_{\nu}$. It would be interesting to know if the syzygies of $I_{U}$ for $U \subset R_{(a, b)}, b>1$ can also be calculated and understood from the generators of the ideal $I_{X}$ of the basepoints of $U$ as is the case for $b=1$.

5.3. Tensor product surfaces without basepoints. The main theorem in this paper allows us to describe the syzygies that determine the implicit equation of a map given by 4 generically chosen forms of bidegree $(a, 1)$ that vanish at a generic set of points in $\mathbb{P}^{1} \times \mathbb{P}^{1}$. The techniques that we used to prove the main theorem depended on understanding the generators of the $k[s, t]$-module $M=\bigoplus_{i=0}^{\infty}\left(I_{X}\right)_{(i, 1)}$ and using the description of $\left(I_{X}\right)_{(a, 1)}$ given by (2) from Section 3. For the case of basepoints, $M=\left\langle g_{1}, g_{2}\right\rangle$. If $X=\emptyset$ then $M=\langle u, v\rangle$. In the proof of the main theorem, we may substitute $g_{1}, g_{2}$ for the complete intersection $u, v$ and the proof will still be valid. We obtain

Theorem 5.6. Let $U=\left\{f_{0}, \ldots, f_{3}\right\}$ be a basepoint free, generic, 4-dimensional vector subspace of $R_{(a, 1)}=H^{0}(a, 1)$ and $\lambda_{U}: \mathbb{P}^{1} \times \mathbb{P}^{1} \rightarrow \mathbb{P}^{3}$ the regular map determined by $U$. Then the first map of the approximation complex $\mathcal{Z}$ in bidegree $\nu=(2 a-1,0)$, is determined by two syzygies of $\left(f_{0}, \ldots, f_{3}\right)$ in bidegree $(a, 0)$.

A careful study of the implicitization of basepoint free tensor product surfaces of bidegree $(2,1)$ using syzygies was done by Schenck, Seceleanu and Validashti [14]. Theorem 5.6 recovers their results for the case that $I_{U}$ has no linear syzygies and extends them to bidegree $(a, 1)$.

5.4. Overview of implicitization methods and examples. As mentioned in the introduction, we may use other methods like Gröbner bases and resultants to find implicit equations of parameterized surfaces. One way to use Gröbner bases is to compute the elimination ideal

$$
J=\left\langle X-f_{0}, Y-f_{1}, Z-f_{2}, W-f_{3}\right\rangle \cap S .
$$


Resultants are used for basepoint free parameterizations of the form $\lambda: \mathbb{P}^{2} \rightarrow \mathbb{P}^{3}$. In this case $H$ is computed by

$$
\operatorname{Res}\left(f_{0}-X f_{3}, f_{1}-Y f_{3}, f_{2}-Z f_{3}\right)=H(X, Y, Z, 1)^{\operatorname{deg} \lambda} .
$$

In the following examples we will compute $H$ using Gröbner bases and the complex $\mathcal{Z}_{\nu}$. For further examples that show the advantages of implicitization using syzygies over other methods, we refer the reader to the work of Botbol and Dickenstein [3](Section 5). The Macaulay2 code to perform the examples that follow is available at https://github.com/emduart2.

Example 5.7. We let $X$ be the set of points in $\mathbb{P}^{1} \times \mathbb{P}^{1}$ in Example 5.2, where $I_{X}=$ $(s, u) \cap(t, v)$. We fix the bidegree $(a, b)=(8,1)$ and let $U \subset\left(I_{X}\right)_{(8,1)}$ be given by

$$
\begin{aligned}
& U=\left\{3 s^{6} t^{2} u+s^{8} v+7 s^{4} t^{4} v-s^{3} t^{5} v, t^{8} u+5 s^{6} t^{2} v+s^{2} t^{6} v,\right. \\
& \left.s^{7} t u+11 s^{5} t^{3} u+s^{2} t^{6} u-s t^{7} u,-s^{4} t^{4} u+s^{3} t^{5} u+s t^{7} u+s^{7} t v+19 s^{5} t^{3} v+s t^{7} v\right\} .
\end{aligned}
$$

The coefficient matrix of $U$ with respect to a monomial basis for $\left(I_{X}\right)_{(8,1)}$ is generic. We use two algorithms in Macaulay2 to compute the first map $d_{1}:\left(\mathcal{Z}_{\nu}\right)_{1} \rightarrow\left(\mathcal{Z}_{\nu}\right)_{0}$ of $\mathcal{Z}_{\nu}$, we refer to these as Algorithm 1 and Algorithm 2. Algorithm 1 computes the minimal syzygies of $I_{U}$, then it selects the two syzygies in bidegree $(7,0)$. Using Theorem 1.1, we know these syzygies are free. Then it bumps up these two syzygies to bidegree $\nu=(2 a-1, b-1)=$ $(15,0)$ by multiplying times the monomials in a basis for $R_{(8,0)}$. Finally it finds $d_{1}$ by applying the Koszul map in the variables $(X, Y, Z, W)$. With this method it takes 0.133 seconds to compute $d_{1}$. Algorithm 2 directly computes a basis for the syzygies of $I_{U}$ and then uses the command super basis $(\{3 * a-1,2 * b-1\}$, image syz Iu) to find a basis of the syzygies in bidegree $\nu=(2 a-1, b-1)$. Finally it proceeds to set up $d_{1}$ as before. With this method the computation of $d_{1}$ takes 1.67 seconds. Notice the speed boost in Algorithm 1 that is obtained from knowing the structure of the syzygies that determine $d_{1}$, in particular we know the exact degrees of the syzygies of $I_{U}$ and that they span a free module. If we use the eliminate command in Macaulay2 to compute the elimination ideal $J$ from equation (7), the computation of the implicit equation takes 149.178 seconds. For this example, $d_{1}$ is a $16 \times 18$ matrix, the implicit equation $H$ of $X_{U}$ has degree 14 and it contains 115 terms.

Example 5.8. Take $U$ to be a random generic 4-dimensional vector subspace of $R_{(20,1)}$, then $U$ is basepoint free. This example can be generated in Macaualay2 by finding a basis of $R_{(20,1)}$ using super basis $(\{20,1\}, \mathrm{R})$ and then multiplying this basis times a random matrix $C$ of coefficients of the correct size. For this choice of $U$, Algorithm 1 takes 0.751 seconds to compute $d_{1}:\left(\mathcal{Z}_{\nu}\right)_{1} \rightarrow\left(\mathcal{Z}_{\nu}\right)_{0}$ and Algorithm 2 takes 180 seconds. The eliminate 
ideal command did not finish the computation in at least 120 minutes and therefore was aborted. In this example, $d_{1}$ is a $40 \times 40$ matrix and $H$ has degree 40 .

Acknoledgements The author would like to thank Hal Schenck for useful discussions and suggestions as well as the referee for the helpful comments that improved the exposition of this work. Evidence for this work was provided by many computations done using Macaulay2, by Dan Grayson and Mike Stillman. Macaulay2 is freely available at http://www.math.uiuc.edu/Macaulay2/ and scripts to perform the computations are available at https://github.com/emduart2.

\section{REFERENCES}

[1] Botвol, N. The implicit equation of a multigraded hypersurface. J. Algebra 348 (2011), 381-401.

[2] Botbol, N., Busé, L., And Chardin, M. Fitting ideals and multiple points of surface parameterizations. J. Algebra 420 (2014), 486-508.

[3] Botbol, N., And Dickenstein, A. Implicitization of rational hypersurfaces via linear syzygies: a practical overview. J. Symbolic Comput. 74 (2016), 493-512.

[4] Busé, L., ANd LuU BA, T. The surface/surface intersection problem by means of matrix based representations. Comput. Aided Geom. Design 29, 8 (2012), 579-598.

[5] Chardin, M. Implicitization using approximation complexes. In Algebraic geometry and geometric modeling, Math. Vis. Springer, Berlin, 2006, pp. 23-35.

[6] Chen, F., Cox, D., And Liu, Y. The $\mu$-basis and implicitization of a rational parametric surface. $J$. Symbolic Comput. 39, 6 (2005), 689-706.

[7] Chen, F., Zheng, J., And Sederberg, T. W. The mu-basis of a rational ruled surface. Comput. Aided Geom. Design 18, 1 (2001), 61-72.

[8] Cox, D. Curves, surfaces, and syzygies. In Topics in algebraic geometry and geometric modeling, vol. 334 of Contemp. Math. Amer. Math. Soc., Providence, RI, 2003, pp. 131-150.

[9] Dонм, M. Implicitization of rational ruled surfaces with $\mu$-bases. J. Symbolic Comput. 44, 5 (2009), 479-489.

[10] Duarte, E., And Schenck, H. Tensor product surfaces and linear syzygies. Proceedings of the American Mathematical Society 144, 1 (2016), 65-72.

[11] Giuffrida, S., Maggioni, R., And Ragusa, A. On the postulation of 0-dimensional subschemes on a smooth quadric. Pacific J. Math. 155, 2 (1992), 251-282.

[12] Guardo, E., And Van Tuyl, A. Arithmetically Cohen-Macaulay Sets of Points in $\mathbb{P}^{1} \times \mathbb{P}^{1}$. Birkhäuser, 2015 .

[13] Herzog, J., Simis, A., And Vasconcelos, W. V. Approximation complexes of blowing-up rings. Journal of Algebra 74, 2 (1982), 466-493.

[14] Schenck, H., Seceleanu, A., And Validashti, J. Syzygies and singularities of tensor product surfaces of bidegree (2,1). Mathematics of Computation 83, 287 (2014), 1337-1372. 
[15] Sederberg, T. W., And Chen, F. Implicitization using moving curves and surfaces. In Proceedings of the 22nd annual conference on Computer graphics and interactive techniques (1995), ACM, pp. 301308.

[16] Van TuYL, A. The border of the Hilbert function of a set of points in $\mathbb{P}^{n_{1}} \times \cdots \times \mathbb{P}^{n_{k}}$. Journal of Pure and Applied Algebra 176, 2 (2002), 223-247.

[17] Van Tuyl, A. The defining ideal of a set of points in multi-projective space. Journal of the London Mathematical Society 72, 1 (2005), 73-90.

Department of mathematics, University of Illinois, Urbana, IL 61801

E-mail address: emduart2@illinois.edu 